\section{Blocking CCR5}

CCR5 is a chemokine receptor that also serves as a co-receptor for HIV-1 gp120 and is essential for HIV-1 entry into some host cells. CCR5 is a class-A G proteincoupled receptor (GPCR) with seven transmembrane segments linked by three extracellular and three intracellular loops. Chemokine ligands bind the N-terminal flexible region of CCR5 and extracellular loop 2, regions that are also involved in HIV-1 gp120 binding. The small-molecule drug maraviroc acts as an allosteric CCR5 inhibitor and has been

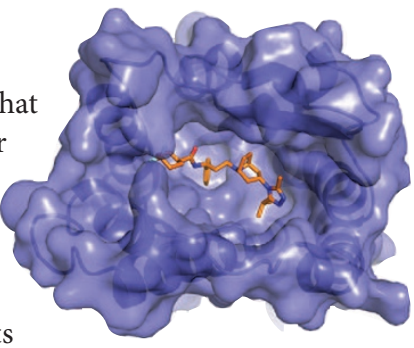
approved to treat HIV-1 infection.

More specifically, maraviroc acts an inverse agonist of CCR5, indicating that the drug stabilizes an inactive conformation of the receptor. Wu and colleagues have now solved the crystal structure of human CCR5 in complex with maraviroc at 2.7- $\AA$ resolution, revealing the structural basis for maraviroc's inhibitory properties. In the structure, maraviroc binds a deep pocket formed by residues from six of the receptor's seven transmembrane helices, without contacting the extracellular loops. Although this binding site does not overlap with the major recognition site for chemokine ligands, it involves regions within the transmembrane domain required for CCR5 activation. In fact, CCR5 seems to be held in an inactive conformation in the complex with maraviroc, as implied by two observations. First, two conserved aromatic residues involved in allosteric communication between the ligand pocket and cytoplasmic regions of GPCRs, Trp248 and Tyr244, assume conformations similar to those adopted in the structures of inactive class-A GPCRs. In particular, Trp248 is locked in a hydrophobic interaction with the phenyl group of maraviroc, and this would prevent the residue's movement to an activestate conformation. In addition, helix VI is closely packed with the other helices at the intracellular side, a conformation that would prevent binding of $\mathrm{G}$ proteins. Together, these features explain how maraviroc allosterically inhibits chemokine signaling and viral infection. (Science doi:10.1126/science.1241475, 12 September 2013)

IC

\section{Dictating histone occupancy}

Nucleosome occupancy within chromatin can affect transcription in a number of ways, from regulating access to transcription factors to posing a barrier to RNA-polymerase elongation. Spt6 is one of multiple histone chaperones involved in the intricate balance between nucleosome deposition and eviction that occurs during transcription. Previous studies have shown that loss of Spt6 results in preferential reduction of nucleosome occupancy on highly transcribed genes, and that the resultant loss of histones can also derepress certain promoters. In a recent study, Bentley and colleagues investigated how Spt6 affects histones and RNA polymerase II (Pol II) occupancy genome wide in the budding yeast Saccharomyces cerevisiae, using a rapidly inactivated Spt6-degron mutant. The key finding is the observation that, upon spt6 inactivation, histone loss-particularly toward the $5^{\prime}$ end of genes-was greater at genes with closed promoters than at those with open promoters, within a subset of genes exhibiting similar Pol II occupancy. Promoter-swap experiments confirmed that histone occupancy within the bodies of genes was determined by the promoters themselves; thus, promoters could confer occupancy levels associated with their respective endogenous genes to unrelated downstream sequences. The precise mechanism by which promoters dictate the level of downstream histone occupancy remains to be determined but probably involves factors such as polymerase density and rates of initiation and/or elongation. Indeed, the authors suggest that closed promoters, which contain TATA boxes and are associated with transcriptional bursting and noisy expression, might be more effective at evicting nucleosomes. Regardless of the ultimate mechanism, the study by Bentley and colleagues uncovered an interesting new element of chromatin biology, which should stimulate further investigations of gene-specific nucleosome dynamics. (EMBO J. doi:10.1038/emboj.2013.194, 6 September 2013) $S L$

\section{RNP-based transcript classification}

Long noncoding RNAs (IncRNAs) and mRNAs share many features, including $5^{\prime}$-methylguanosine caps and poly(A) tails, and both are transcribed by RNA polymerase II (Pol II). However, most IncRNAs are retained in the nucleus, whereas almost all mRNAs are exported to the cytoplasm, suggesting that their RNA maturation paths diverge at some point. All transcripts interact with a series of protein factors during their maturation, thus forming ribonucleoprotein particles (RNPs). Tuck and Tollervey therefore analyzed the in vivo, transcriptome-wide targets of 13 RNA-processing, export and turnover factors in budding yeast, using the cross-linking and analysis of CDNA (CRAC) technique, to produce a transcriptome-wide survey of RNP composition. Comparison of the maturation pathways of mRNAs and the stable unannotated transcript (SUT) and cryptic unstable transcript (CUT) classes of IncRNAs revealed that transcript fate is largely determined during $3^{\prime}$-end formation. Whereas mRNAs and SUTs carried the hallmarks of cleavage and polyadenylation, these were absent from CUTs, which undergo rapid degradation in the nucleus. The RNP composition of SUTs overlapped significantly with that of mRNAs, with some SUTs being retained in the nucleus while others were exported to the cytoplasm. Moreover, about $10 \%$ of mRNAs behaved like IncRNAs, being retained and degraded in the nucleus. The extensive heterogeneity in mRNP composition, combined with gene ontology-term analyses, led the authors to suggest that an mRNA's tailored mRNP composition may be linked to the function of the encoded protein. Nuclear surveillance factors were found to bind promoter-proximal IncRNAs generated by early transcription termination, which occurred to some extent for most mRNAs and could reflect a checkpoint in Pol II transcription. Whether these early-terminating transcripts are functional remains to be determined. This transcriptome-wide survey of RNP composition allows for an RNP-based classification of transcripts, which reflects their regulation and possible function. (Cel/ 154, 996-1009, 2013) 